\title{
Intergroup Rhabdomyosarcoma Group II
}

National Cancer Institute

\section{Source}

National Cancer Institute. Intergroup Rhabdomyosarcoma Group II. NCI Thesaurus.

Code C148015.

Total gross resection with regional disease spread. 